\title{
Pourquoi l'interdiction des visites dans les hôpitaux durant l'actuelle pandémie de COVID-19 devrait être assouplie
}

\author{
Laveena Munshi MD, Gerald Evans MD, Fahad Razak MD MSc
}

— Citation : CMAJ 2021 January 25;193:E135-7. doi : 10.1503/cmaj.202636-f; diffusion hâtive le 18 décembre 2020

Voir la version anglaise de l'article ici : www.cmaj.ca/lookup/doi/10.1503/cmaj.202636

$\mathbf{P}$

our tenter de limiter la transmission galopante du coronavirus du syndrome respiratoire aigu sévère 2 (SRASCoV-2), les hôpitaux canadiens ont adopté des politiques d'interdiction des visites durant la première vague de la pandémie. Des politiques systématiques et quasi complètes de restriction des visites ont été appliquées par les 35 hôpitaux interrogés dans le cadre d'une étude pancanadienne ${ }^{1}$. Ces politiques avaient pour but d'équilibrer les intérêts des patients et de leurs proches aidants et la responsabilité utilitaire de la société de réduire la transmission vers les hôpitaux et en leur sein, avec comme objectif secondaire de ménager l'équipement de protection individuelle (EPI) alors qu'une pénurie menaçait. Au cœur d'une seconde vague, il est prudent de se demander si ces restrictions doivent rester en vigueur pour la prévention et le contrôle des infections ( $\mathrm{PCl}$ ) et comme principe de précaution, ou s'il faut les assouplir pour prévenir les préjudices qu'elles causent aux patients et aux familles.

L'interdiction des visites dans les hôpitaux n'est pas l'apanage de la pandémie de maladie à coronavirus 2019 (COVID19) et est appliquée depuis plusieurs décennies. Les leçons tirées des épisodes de grippe saisonnière laissent croire que la prévention des éclosions de virus respiratoires dans les unités hospitalières de soins actifs est indispensable pour que les hôpitaux puissent continuer de fournir à tous les soins requis². Toutefois, un assouplissement progressif de ces restrictions a débuté après que l'on ait accumulé des preuves de l'effet bénéfique de la présence des proches ou des personnes de soutien désignées sur les patients hospitalisés ${ }^{3,4}$. Avant la pandémie de COVID-19, une revue systématique ayant évalué l'incidence des politiques de visites autorisées $\mathrm{c}$. restreintes chez les patients adultes dans le contexte des soins intensifs (USI) n'a fait état d'aucune augmentation de la transmission des infections ou des complications septiques en lien avec des politiques plus souples ${ }^{3}$. Une revue de 2020 (avant la pandémie) a observé que 73\% des hôpitaux avaient adopté des politiques d'accommodement en regard des visites, comparativement à seulement $32 \%$ en $2015^{1}$.

\section{POINTS CLÉS}

- Des politiques d'interdiction des visites ont été adoptées au Canada pendant la première vague de la pandémie pour tenter de réduire l'introduction et la propagation du coronavirus du syndrome respiratoire aigu sévère 2 (SRAS-CoV-2) dans les hôpitaux, et pour réserver l'équipement de protection individuelle (EPI) aux travailleurs de la santé.

- La littérature scientifique disponible n'attribue pas de rôle majeur aux membres des familles et aux partenaires de soins désignés dans la transmission du SRAS-CoV-2 dans les hôpitaux.

- Beaucoup de données ont montré que les membres des familles ou partenaires de soins désignés sont importants pour la prestation des soins axés sur les patients, particulièrement en ce qui concerne la défense de leurs intérêts, l'alimentation, la mobilité, l'orientation, le soutien émotionnel dans un contexte de délire, d'atteinte cognitive, de barrière linguistique, de soins de fin de vie, à l'unité des naissances et lors de transferts vers les soins intensifs.

- Selon nous, au cours de l'actuelle vague de la pandémie de maladie à coronavirus 2019 (COVID-19), les hôpitaux devraient adopter des politiques plus souples à l'égard des visites, avec une utilisation stricte de l'EPI et une surveillance adéquate, en portant davantage attention à la prévalence communautaire de la COVID-19 et au statut d'immunisation prévisible.

À l'heure actuelle, on dispose de preuves limitées pour appuyer un quelconque rôle majeur des visiteurs dans la transmission intrahospitalière de la COVID-19. Au début de la pandémie, avant l'application à grande échelle des mesures de santé publique et de $\mathrm{PCl}$, une revue systématique sur les acquisitions nosocomiales de la COVID-19 à partir de séries de cas de Wuhan, en Chine, n'a fait état que de $2 \%$ provenant de personnes autres que le personnel soignant (c'est-à-dire, de visiteurs) $)^{5}$. Une étude plus récente sur 9149 patients hospitalisés aux États-Unis entre le 7 mars et le 30 mai 2020 a constaté des taux faibles de COVID-19 acquise à l'hôpital' . Seulement 1,7\% des patients (12/697) atteints de COVID19 présentaient des résultats positifs au test de dépistage 3 jours 
après leur admission ou dans les 14 jours suivant leur congé et 10 de ces 12 patients étaient probablement des cas d'identification tardive d'une acquisition communautaire. Un seul a été associé à un visiteur présymptomatique avant l'application des restrictions. Les taux faibles ne peuvent pas être attribuables uniquement aux restrictions imposées aux visiteurs parce que ces politiques ont été adoptées alors que l'étude suivait déjà son cours.

Les politiques systématiques à grande échelle de restriction des visites sont associées à plusieurs facteurs de risque pour les patients, les familles et les professionnels de la santéz,4,7-10. Même si le terme visiteur englobe généralement toute personne ayant un lien avec un patient, il importe de distinguer les visiteurs en général des «membres de la famille » ou "des personnes de soutien désignées » (aussi appelés partenaires de soins désignés). L'importance des membres de la famille ou des partenaires de soins désignés est de plus en plus reconnue dans la prestation des soins axés sur les patients (p. ex., aide pour l'alimentation, la mobilité, l'hygiène, l'orientation et le soutien émotionnel). On a démontré que des politiques de visites plus souples peuvent améliorer la communication et créer un lien de confiance entre les familles et le personnel soignant, surtout en présence de barrières linguistiques ${ }^{7}$. Les membres de la famille sont essentiels à la communication tout au long du continuum de soins, et plus particulièrement si un patient devient inapte ils sont là pour s'exprimer en son nom.

Selon des recherches préliminaires, le délire est fréquent dans les formes graves et aiguës de COVID-19 ${ }^{8}$. Les patients délirants séjournent plus longtemps à l'hôpital et ont un risque de mortalité plus élevé. Dans le contexte des USI, la participation des familles et les politiques de visite plus souples ont été associées à une réduction du délire et à une amélioration du rétablissement psychologique à long terme ${ }^{3}$. En général, les interventions de soins axés sur la famille permettent d'abréger le séjour aux USI et d'améliorer l'expérience des patients, de même que la santé mentale des patients et de leurs proches ${ }^{4}$.

Une récente étude d'observation a mesuré l'effet de la restriction des visites sur l'expérience postopératoire de patients opérés durant la pandémie de COVID-199. Les patients soumis à une restriction des visites ont tardé à recevoir des médicaments, ont vécu plus d'isolement social, ont eu de la difficulté à se lever du lit et étaient plus susceptibles de voir leurs préférences non respectées au moment du congé, comparativement aux patients exemptés de la restriction des visites avant son instauration.

Les données sont limitées quant à l'incidence des politiques de restriction des visites sur les familles. Toutefois, une étude d'observation de 2017 a révélé que l'incapacité d'un patient agonisant de faire ses adieux à ses proches était associée à un deuil plus difficile pour la famille ${ }^{10}$. Mourir seul cause de la détresse et de la confusion chez les patients et une détresse psychologique chez leurs proches. Les travailleurs de la santé ont fait état d'un désarroi substantiel associé au fait de « se substituer » aux familles auprès des patients agonisants ${ }^{11}$. Les participants à une étude auprès de professionnels de la santé des USI en France entre avril et mai 2020 ont exprimé un profond regret et des symptômes d'anxiété ou de dépression en lien avec la restriction des visites dans le contexte de la COVID-1912.
Comment équilibrer ces risques à mesure que la pandémie évolue? Pour assurer l'application de mesures de $\mathrm{PCl}$ adéquates alors que la prévalence communautaire de la COVID-19 est en hausse, certaines restrictions sont requises. L'augmentation du nombre de visiteurs rendra plus difficile le maintien de la distanciation physique adéquate (p. ex., dans les ascenseurs et les halls d'entrée). Des éclosions nosocomiales ont été rapportées lors des 2 vagues de la pandémie au Canada. Certaines peuvent avoir été causées par des travailleurs de la santé asymptomatiques infectés dans la communauté ayant utilisé des espaces communs lors des pauses et des repas, alors que d'autres ont pu avoir été causées par des visiteurs asymptomatiques trop peu formés en matière d'EPI ou ayant omis de déclarer leurs symptômes lors du questionnaire à l'arrivée.

Durant la seconde vague de la pandémie, de nombreux établissements au Canada ont commencé à adopter une politique moins restrictive, reconnaissant l'apport des familles et des partenaires de soins désignés, après avoir tenu compte de leurs expériences de la première vague. Parmi les établissements qui devraient faire des exceptions pour les membres des familles ou les partenaires de soins désignés, mentionnons les centres de soins de longue durée, les unités de soins palliatifs, les USI, les unités des naissances et les services de chirurgie. Les patients transférés aux soins intensifs ou aux soins palliatifs et les patients qui souffrent de délire, d'une atteinte cognitive, en situation de barrière linguistique ou atteints de graves maladies psychiatriques, pourraient aussi bénéficier de politiques de visite moins austères.

On ignore quelle est l'incidence des différentes politiques en matière de visites sur le risque d'acquisition de la COVID-19. La prévalence communautaire de l'infection, y compris le taux d'infection asymptomatique, le mode de transmission et la qualité de la recherche des contacts, influera sur les taux de transmission dans les hôpitaux. Toutefois, des mesures de $\mathrm{PCl}$ strictes (par exemple, port du masque, lavage des mains et dépistage à l'arrivée à l'hôpital) et un test rapide de COVID-19 performant pourraient limiter substantiellement le risque de transmission. Il faut recueillir des données pour mieux établir l'équilibre entre ces divers facteurs concurrents. Une variation substantielle de la prévalence de la COVID-19 entre les régions signifie que les risques devraient être évalués à l'échelle régionale ou à l'échelle des hôpitaux et les politiques de visite devraient être adaptées en conséquence. Les étapes propices à une réduction du risque de transmission incluent mieux dépister et renseigner les visiteurs, les limiter à un nombre précis de membres de la famille ou de partenaires de soins désignés par patient, restreindre la durée des visites, utiliser sélectivement les tests de dépistage cliniques (si possible), retracer les contacts par région (selon le cas) et assurer le port d'un EPI approprié pour les visiteurs.

La sécurité des visites repose sur le signalement précis et honnête de tout symptôme de la part des visiteurs, de leur accès à de l'EPI et de leur respect des consignes en vigueur sur le port de celui-ci. Un sondage de 2018 auprès de visiteurs de patients porteurs d'une infection à Clostridium difficile a révélé que le personnel infirmier joue un rôle central dans la sensibilisation des visiteurs à la bonne utilisation de l'EPI ${ }^{13}$. Il faudrait assigner du personnel additionnel et mieux renseigner les visiteurs pour que 
l'EPI soit utilisé de manière appropriée. Étant donné les difficultés plus grandes d'assurer une utilisation appropriée de l'EPI pour les visiteurs de patients atteints de COVID-19, il pourrait falloir restreindre davantage les visites pour ce sous-groupe de patients.

Si les visiteurs doivent être gardés à l'écart, l'accès facile à un système de vidéoconférence peut réduire les risques inhérents à l'isolement des patients. Une étude qui évaluait la faisabilité et l'acceptabilité des communications électroniques pour la tenue de réunions familiales dans le contexte des soins palliatifs pendant la pandémie a révélé que les vidéoconférences pouvaient être planifiées avec succès dans $97 \%$ des cas, et que $80 \%$ des membres des familles se sentaient à l'aise de poser leurs questions et de s'exprimer au cours de telles rencontres ${ }^{14}$. Toutefois, la vidéoconférence requiert l'accès à des appareils et l'aide du personnel soignant, ce qui alourdit leur tâche et ne permet pas les conversations impromptues. Or, la situation est délicate lorsque la discussion porte sur les volontés d'un patient en matière de soins de fin de vie, et rien ne prouve que les renseignements d'un patient soient dans ce cas partagés exclusivement avec des gens choisis par le patient. De plus, l'utilisation des appareils mobiles pourrait être une cause de transmission virale s'ils sont contaminés et par conséquent, les mesures de $\mathrm{PCl}$ s'appliquent pour la désinfection des appareils ${ }^{15}$. Dans la mesure du possible, certains centres ont utilisé des espaces extérieurs pour faciliter les visites entre les patients et leurs proches.

La littérature scientifique existante n'appuie aucun scénario selon lequel une restriction complète des visites pour tous les types de patients des hôpitaux serait requise. Même si les données ne sont pas concluantes, nous croyons que les politiques de restriction des visites pourraient faire plus de mal que de bien dans certains contextes, et il y a des cas précis où les visites à l'hôpital devraient être autorisées moyennant une utilisation rigoureuse de l'EPI et une surveillance adéquate, en portant une attention particulière à la prévalence communautaire de la COVID-19 et au statut d'immunisation communautaire prévisible. Une étude canadienne est en cours pour mesurer l'incidence de la pandémie sur les politiques de visite dans les hôpitaux canadiens (https://cumming.ucalgary.ca/research/ calgary-critical-care-research-network/research/COVID-19).

\section{Références}

1. Re-integration of family caregivers as essential partners in care in a time of COVID-19. Ottawa: Canadian Foundation for Healthcare Improvement; 2020. Accessible ici : www.cfhi-fcass.ca/about/news-and-stories/news-detail/2020 /07/08/re-integration-of-family-caregivers-as-essential-partners-in-care-in-a-time -of-covid-19 (consulté le 17 oct. 2020).

2. Salgado CD, Farr BM, Hall KK, et al. Influenza in the acute hospital setting. Lancet Infect Dis 2002;2:145-55.

3. Nasser Junior AP, Besen BAMP, Robinson CC, et al. Flexible versus restrictive visiting policies in ICUs: a systematic review and meta-analysis. Crit Care Med 2018;46:1175-80.

4. Goldfarb MJ, Bibas L, Bartlett V, et al. Outcomes of patient- and family-centred care interventions in the ICU: a systematic review and meta-analysis. Crit Care Med 2017;45:1751-61.

5. Zhou Q, Gao Y, Wang X, et al. Nosocomial infections among patients with COVID-19, SARS and MERS: a rapid review and meta-analysis. Ann Transl Med 2020;8:629.
6. Rhee C, Baker M, Vaidya V, et al. Incidence of nosocomial COVID-19 in patients hospitalized at a large US academic medical center. JAMA Netw Open 2020;3: e2020498.

7. Hurst $\mathrm{H}$, Griffiths J, Hunt C, et al. A realist evaluation of the implementation of open visiting in an acute care setting for older people. BMC Health Serv Res 2019;19:867.

8. Helms J, Kremer S, Merdji H, et al. Delirium and encephalopathy in severe COVID-19: a cohort analysis of ICU patients. Crit Care 2020;24:491.

9. Zeh RD, Santry H, Monsour C, et al. Impact of visitor restriction rules on the postoperative experience of COVID-19 negative patients undergoing surgery. Surgery 2020;168-770-76.

10. Otani H, Yoshida S, Morita T, et al. Meaningful communication before death, but not present at the time of death itself, is associated with better outcomes on measures of depression and complicated grief among bereaved family members of cancer patients. J Pain Symptom Manage 2017;54:273-79.

11. Najeeb U. 2020. COVID-19 reflections: Phone call [blog]. CMAJ 2020 July 3. Accessible ici : http://cmajblogs.com/phone-call/ (consulté le 18 nov. 2020).

12. Azoulay E, Cariou A, Bruneel F, et al. Symptoms of anxiety, depression and peritraumatic dissocation in critical care clinicians managing COVID-19 patients: a cross-sectional study. Am J Respir Crit Care Med 2020;202:1388-98.

13. Seibert G, Ewers T, Barker A, et al. What do visitors know and how do they feel about contact precautions? Am J Infect Control 2018;46:115-7.

14. Kuntz JG, Kavalieratos D, Esper G, et al. Feasibility and acceptability of inpatient palliative care e-family meetings during COVID-19 pandemic. J Pain Symptom Manage 2020;60:e28.

15. Brady RR, Verran J, Damani NN, et al. Review of mobile communication devices as potential reservoirs of nosocomial pathogens. J Hosp Infect 2009;71:295-300.

Intérêts concurrents : Fahad Razak est à l'emploi de Santé Ontario. Aucun autre intérêt concurrent n'a été déclaré.

Cet article a été révisé par des pairs.

Affiliations : Division interdépartementale de médecine des soins intensifs, Département de médecine (Munshi), Système de santé Sinaï et Réseau universitaire de santé, Université de Toronto; Institut de gestion, d'évaluation et de politiques de santé (Munshi, Razak), Université de Toronto, Toronto, Ont.; Ontario COVID-19 Science Advisory Table (Munshi, Evans, Razak); Division d'infectiologie, Département de médecine (Evans), Centre des sciences de la santé de Kingston, Université Queen's, Kingston, Ont.; Service de médecine (Razak), Hôpital St. Michael, Unity Health; Division de médecine interne générale, Département de médecine (Razak), Université de Toronto; Institut du savoir Li Ka Shing (Razak), Hôpital St. Michael, Toronto, Ont.

Collaborateurs : Tous les auteurs ont contribué à la conception du manuscrit et à l'interprétation de la littérature scientifique actuelle. Laveena Munshi a rédigé la première ébauche. Tous les auteurs ont contribué aux révisions de l'ébauche du manuscrit, ont donné leur approbation finale pour la version destinée à être publiée et assument l'entière responsabilité de tous les aspects du travail.

Financement : Fahad Razak bénéficie d'une subvention de la chaire Mak Pak Chiu et Mak-Soo Lai Hing en médecine interne générale, Université de Toronto.

Propriété intellectuelle du contenu : Il s'agit d'un article en libre accès distribué conformément aux modalités de la licence Creative Commons Attribution (CC BY-NC-ND 4.0), qui permet l'utilisation, la diffusion et la reproduction dans tout médium à la condition que la publication originale soit adéquatement citée, que l'utilisation se fasse à des fins non commerciales (c.-à-d., recherche ou éducation) et qu'aucune modification ni adaptation n'y soit apportée. Voir : https://creativecommons.org/licenses/by-nc-nd/4.0/deed.fr.

Correspondance : Laveena Munshi, Laveena.munshi@sinaihealth.ca 